\title{
Analysis of trimethylsilyl derivatization products of phosphatidylethanol by gas chromatography-mass spectrometry
}

\author{
Changsuek Yon ${ }^{1}$ and Joong-Soo Han ${ }^{1,2}$ \\ ${ }^{1}$ Institute of Biomedical Science and Department of Biochemistry, \\ College of Medicine, Hanyang University, 17 Haengdang-Dong, \\ Sungdong-Ku, Seoul 133-792, Korea \\ ${ }^{2}$ Corresponding Author: Tel, +82-2-2290-0623; \\ Fax, +82-2-2294-6270; E-mail, jshan@email.hanyang.ac.kr
}

Accepted 13 December 2000

Abbreviations: BSTFA, N,O-bis (trimethylsilyl) trifluoroacetamide; PEt, phosphatidylethanol; PLD, phospholipase D; TMCS, trimethylchlorosilane; TMS, trimethylsilyl

\begin{abstract}
For the detection of rare phospholipid, phosphatidylethanol (PEt), GC-MS analysis method was adopted for the detection of derivatization products of PEt by N,O-bis (trimethylsilyl) trifluroacetamide (BSTFA). A re-structured molecule derived from PEt, ethyl bis (trimethylsilyl)-phosphate was found from search of Wiley database. This molecule can be used as a marker for PEt analysis. The molecular formula was $\mathrm{C}_{8} \mathrm{H}_{23} \mathrm{O}_{4} \mathrm{PSi}_{2}$ and weight of the formula was 270.09.
\end{abstract}

Keywords: GC-MS, phosphatidylethanol, trimethylsilyl derivative, BSTFA

\section{Introduction}

Phospholipase D (PLD) catalyses hydrolysis of the terminal phosphodiester bond of membrane glycerophospholipids, resulting in the formation of phosphatidic acid (PA) and a related head group (Kobayashi and Kanfer, 1987; Exton, 1998; Gomez-Cambronero et al., 1998). For in vitro activity study of this enzyme, short-chain aliphatic alcohols (ethyl or butyl alcohols) are added in the reaction mixture (Shin et al., 1998). PLD produces rare phospholipids, phosphatidylethanol (PEt) and phosphatidylbutanol (PBt) respectively, instead of PA with a unique catalytic activity of transphosphatidylation in the presence of primary alcohol. The reaction products, PEt or PBt are used as markers of in vitro PLD activity in many studies. The main product of PLD reaction, PA, is subsequently converted into lyso-PA (LPA) by phospho- lipase $\mathrm{A}_{2},\left(\mathrm{PLA} \mathrm{A}_{2}\right)$ or DAG by PA-phosphohydrolase.

For the detection of PEt to determine PLD activity, liquid scintillation counting (LSC) method after thin layer chromatography (TLC) development was widely used (Exton, 1998; Oh et al., 2000). Instrumental analysis for the rare phospholipids was not adopted usually because of the characteristics of the rare phospholipids. PEt or PBt did not give satisfactory UV absorption when applied with high performance liquid chromatography (HPLC)UV detector system because they do not have enough UV absorption groups in the molecules. For gas chromatography (GC) analysis, they are too heavy to evaporate in the inlet of GC. Recently, analysis of PEt with various detectors like evaporative light scattering detector (ELSD), fluorescence detector or mass spectrometry (MS) were reported from several research groups (Gunnarsson et al., 1998; Kemken et al., 2000; Park et al., 2000; Varga et al., 2000). To facilitate analysis with GCMS, PEt was derivatized and re-structured by BSTFA. From the reaction mixture, we found 2 trimethylsilyl (TMS) derivatives that could represent PEt.

\section{Materials and Methods}

\section{Chemicals}

1,2-Dipalmitoyl-sn-Glycero-3-Phosphoethanol (sodium salt, PEt) was purchased from Avanti Polar-Lipids, Inc. (Alabaster, AL, USA). N,O-bis (trimethylsilyl) trifluroacetamide (BSTFA) plus 1\% Trimethylchlorosilane (TMCS) was purchased from PIERCE (Rockford, IL, USA). All other reagents and solvents used for the experiments were of analytical grade.

\section{GC-MS analysis of PEt}

GC-MS analysis was performed with HP5-MS capillary column $(0.25 \mathrm{~mm}$ ID, $30 \mathrm{~m}$ length, $0.25 \mu \mathrm{m}$ thickness, Hewlett-Packard) with HP6890 GC and HP5973 Mass detector. Mass spectrum was obtained by electron impact ionization mode, scanning from $50 \mathrm{~m} / \mathrm{z}$ to $500 \mathrm{~m} / \mathrm{z}$. Inlet temperature was $180^{\circ} \mathrm{C}$ and oven temperature program was as follows: an initial holding at $100^{\circ} \mathrm{C}$ for $10 \mathrm{~min}$; raising at a rate of $10^{\circ} \mathrm{C} / \mathrm{min}$ to $170^{\circ} \mathrm{C}$; and a final holding at $270^{\circ} \mathrm{C}$ for $15 \mathrm{~min}$.

\section{Derivatization}

Five miligrams of PEt was dissolved in $1 \mathrm{ml}$ of chloroform and $0.5 \mathrm{ml}$ of BSTFA plus $1 \%$ TMCS was added. 


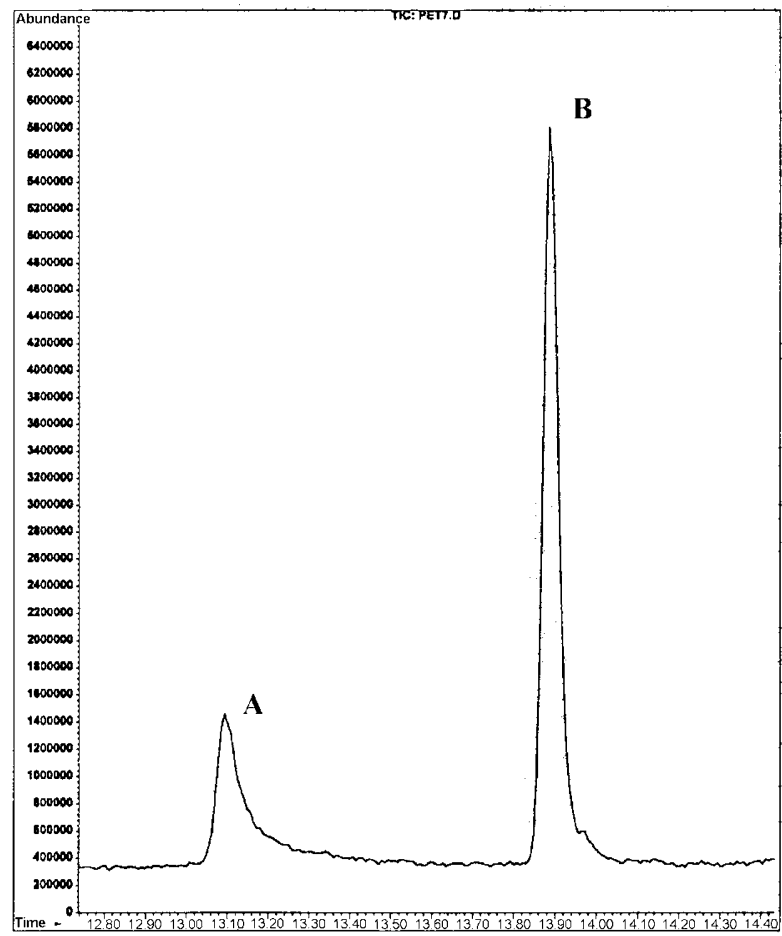

Figure 1. Total ion chromatogram $(\mathrm{TIC})$ of TMS derivatization products of PEt. A: Ethyl bis (trimethylsilyl)-phosphate, B: Tris (trimethylsilyl)phsophate

Derivatization was completed by standing the reaction mixture for $1 \mathrm{~h}$ at room temperature.

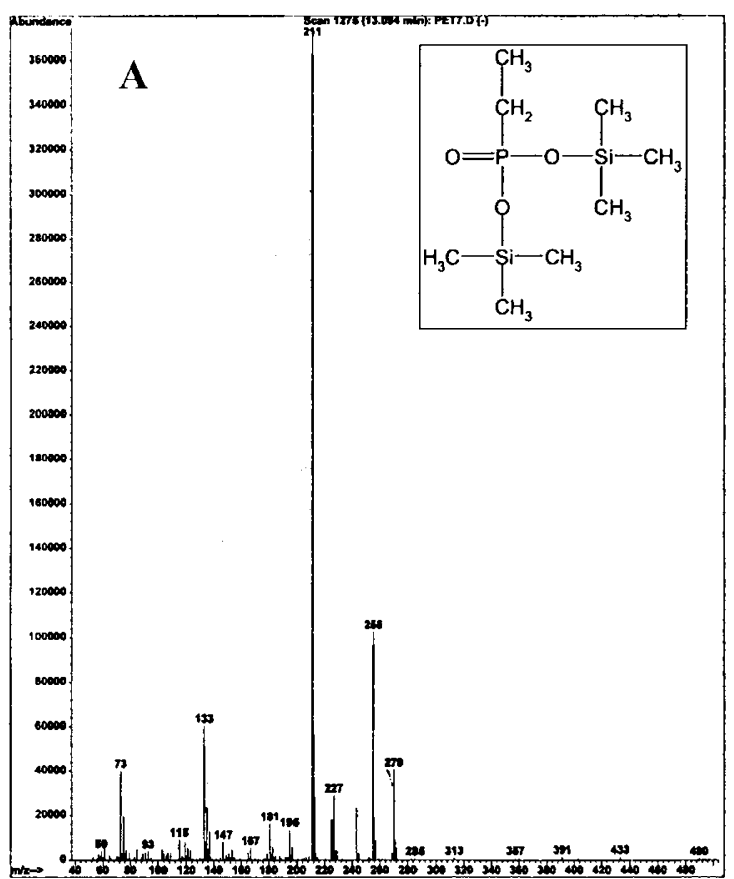

\section{Results}

One microliter of the reaction mixture was injected through the inlet of GC-MS with split ration of $10: 1$. By database search against the separated peaks, two significant molecules, ethyl bis (trimethylsilyl)-phosphate and tris (trimethylsilyl)-phosphate were found. Retention time (RT) of the molecules were $13.1 \mathrm{~min}$ and $13.9 \mathrm{~min}$ respectively (Figure 1). The two molecules were well separated and believed to be re-structured products during TMS derivatization originated from ethylphosphate group of PEt, based on the elucidation of structural characteristics and mass profiles (Figure 2). Match quality of ethyl bis (trimethylsilyl)-phosphate from Wiley database search was 80 , and weight and molecular structure of the formula were 270.09 and $\mathrm{C}_{8} \mathrm{H}_{23} \mathrm{O}_{4} \mathrm{PSi}_{2}$ respectively. Base ion of ethyl bis (trimethylsilyl)-phosphate is $\mathrm{m} / \mathrm{z} 211$. Match quality of tris (trimethylsilyl)-phosphate from Wiley database search was 96 , and weight and molecular structure of the formula were 314.10 and $\mathrm{C}_{9} \mathrm{H}_{27} \mathrm{O}_{4} \mathrm{PSi}_{3}$ respectively. Base ion of this molecule is $\mathrm{m} / \mathrm{z}$ 299. TMS ester of the acyl group in PEt molecule, palmitic acid, was found and identified also (data not shown). With selected ion monitoring (SIM) mode at $\mathrm{m}$ / $z 211$ and 299, the detection limit can be lowered.

\section{Discussion}

Conventional PEt detection method is convenient to use

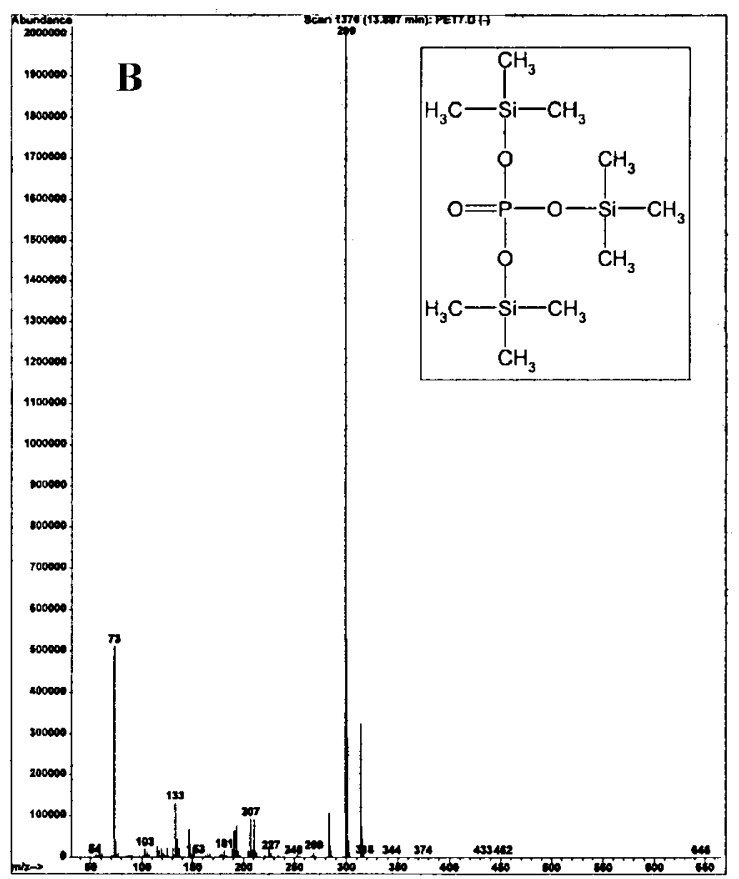

Figure 2. GC-MS profiles and molecular structure of TMS derivatization products. A: Mass pattern and molecular structure of ethyl bis (trimethylsilyl)-phosphate, B: Mass pattern and molecular structure of tris (trimethylsilyl)-phsophate 
in most labs. and gives sufficient sensitivity for the detection of PEt and determine the activity of PLD in cultured cells, but has several problems to solve. Quantitative analysis of PEt and application of various samples such as blood or tissues are difficult with the conventional PEt detection method. HPLC analysis can be a good substitution for the conventional method because it can detect intact PEt molecules. But with structural characteristics of PEt, general UV detectors can not be used for the analysis. TMS derivatization of PEt and GC analysis provide several advantages compared to HPLC method. PEt contents in biological samples are usually very low, nmole to pmole orders, and sensitive analysis methods are required. In general, GC is thousands of folds more sensitive than HPLC method. PEt generated in the cell is comprised of several molecular species in the fatty acid groups (Gunnarsson, 1998). Because ethyl bis (trimethylsilyl)-phosphate and tris (trimethylsilyl)-phosphate represent constant part of PEt, deviation caused by variety of molecular species in the acyl group can be compensated.

Among the two TMS derivatives, ethyl bis (trimethylsilyl)-phosphate can be a marker molecule in GC-MS analysis of PEt because non-specific tris (trimethylsilyl)phosphate originated from other cellular components can be generated during derivatization of biological samples. With data from application study of biological samples and more specified experiments for TMS derivatization, this new method of analysis for the detection of PEt in biological samples can be successfully substituted for the conventional method.

\section{Acknowledgement}

This work was supported by a grant (2000) from Hanyang University and Dong-A Pharmaceutical. We thank Food Safety Research Center and Food R\&D Center of Cheiljedang Corp. for providing GC-MS.

\section{References}

Exton, J. (1998) Phospholipase D. Biochim. Biophys. Acta. 1436: $105-115$

Gomez-Cambronero, J. and Jeire, P. (1998) Phospholipase D: A Novel Major Player in Signal Transduction. Cell Signal. 10: 387-397

Gunnarsson, T., Karlsson, A., Hansson, P., Johnson, G., Alling, C. and Odham, G. (1998) Determination of phosphatidylethanol in blood from alcoholic males using high-performance liquid chromatography and evaporative light scattering or electrospray mass spectrometric detection. J. Chromatogr. B. 705: 243-249

Kemken, D., Mier, K., Katus, H. A., Richardt, G. and Kurz, T. (2000) A HPLC-fluorescence detection method for determination of cardiac phospholipase $\mathrm{D}$ activity in vitro. Anal. Biochem. 286: 277-281

Kobayashi, M. and Kanfer, J. N. (1987) Phosphatidylethanol formation via transphosphatidylation by rat brain synaptosomal phospholipase D. J. Neurochem. 1597-1603

Oh, T.-W., Kim, J.-H., Lee, K. S. and Han, J.-S. (2000) Major house dust mite allergen, Der pl, activates phospholipase D in human peripheral blood mononuclear cells from allergic patients: Involvement of protein kinase C. Exp. Mol. Med. 32: 67-71

Park, H. S., Lee. S. Y., Kim, Y. H., Kim, J. Y., Lee, S. J. and Choi, M. (2000) Membrane perturbation by mastoparan 7 elicits a broad alteration in lipid composition of L1210 cells. Biochim. Biophys. Acta. 1484: 151-162

Shin, I., Kang, J. H. and Han, J.-S. (1998) Changes of phospholipase D activity in TNF- $\alpha$ and anti-Fas/Apo1 monoclonal antibody induced apoptosis in HL-60 and A20 cells. Exp. Mol. Med. 30: 21-27

Varga, A., Hansson, P., Johnson, G. and Alling, C. (2000) Normalization rate and cellular localization of phosphatidylethanol in whole blood from chronic alcoholics. Clin. Chim. Acta. 299: 141-150 